\title{
Gastric Gangrene Due to Necrotizing Gastritis
}

\author{
Madhumita Mukhopadhyay • Anil K. Saha • \\ Amitava Sarkar • Samarendra N. Tripathi • \\ Swapan Biswas • Debasis Mandal • Swadhin Mukherjee
}

Received: 6 December 2008 / Accepted: 2 January 2009 / Published online: 16 November 2010

(C) Association of Surgeons of India 2010

\begin{abstract}
Gangrene of the stomach is a rare, often fatal disease which may be due to vascular, chemical, mechanical or infectious etiologies. We report a case of gastric gangrene due to necrotizing gastritis in a 50 year old female.
\end{abstract}

Keywords Gastric gangrene - Acute necrotizing gastritis . Management

\section{Introduction}

Of the several causes of gastric necrosis, the rarest is acute necrotizing gastritis which appears to be a variant of phlegmonous gastritis [1]. In acute necrotizing gastritis all four major gastric vessels are patent, but gastric gangrene occurs secondary to a necrobiotic infection [1]. In literature cases of acute necrotizing gastritis have been linked to peptic ulcer disease, gastric outlet obstruction and vagotomy [2,3]. We present a case of gastric gangrene due to acute necrotizing gastritis in a patient in whom no apparent predisposing cause could be found.

\section{Case Report}

A 50 year old lady was admitted to the general surgery emergency with a 24 hours duration of pain in the epigastrium. The pain was severe, continuous, associated with nausea. There was no history of haematemesis or

M. Mukhopadhyay $(\bowtie) \cdot$ A. K. Saha $\cdot$ A. Sarkar $\cdot$ S. N. Tripathi $\cdot$

$\mathrm{S}$. Biswas $\cdot \mathrm{D}$. Mandal $\cdot \mathrm{S}$. Mukherjee

Calcutta National Medical College \& Hospital,

Kolkata, India

e-mail:drmmeettuu@yahoo.co.in vomiting. The past medical history was not significant. She was HIV negative. On examination she was in agonizing pain. The vital signs were, Temperature $101^{\circ} \mathrm{F}$, Pulse $105 / \mathrm{min}$, Respiratory rate $26 / \mathrm{min}$, BP $100 / 70 \mathrm{mmHg}$. Abdominal examination found diffuse tenderness with rigidity on palpation. Bowel sounds were sluggish. Rectal examination was normal. The Ryle's tube showed haemorrhagic foul smelling aspirate. Upright chest $\mathrm{x}$-ray showed no free gas under the diaphragm. Routine hemogram showed leucocytosis. Blood sugar level and serum urea, creatinine levels were within normal limits. After initial resuscitation with intravenous fluids and antibiotics, decision was taken to proceed for an emergency exploratory laparotomy.

On exploration, the peritoneal cavity was filled with haemorrhagic fluid. There was gangrene of the distal 2/3rd of the stomach with intact extrinsic blood supply (Fig. 1). On opening the stomach, it was filled with foul smelling haemorrhagic fluid. There was normal pulsation of the gastric vessels. The esophagus, small intestine, colon and rectum were pink and viable. Distal 2/3rd of the stomach was excised. The duodenal stump was closed. The proximal small gastric stump was closed after insertion of a tube drain. This was followed by a feeding jejusnostomy and the abdomen was closed (Fig. 2). Definitive surgery was not done at this time because the patient's condition was unstable during surgery and secondly there was doubt whether the gangrene will spread further. E. Coli was isolated from the culture of the peritoneal fluid. Histopathological examination of the resected stomach showed haemorrhage and necrosis.

After 6 weeks the patient underwent a second stage operation. A loop of jejunum was anastomosed to the remnant gastric stump. Post operative recovery was uneventful. Barium swallow was performed on the 14 postoperative day (Fig. 3). There was no leak. The patient was discharged home and is currently under follow up. 


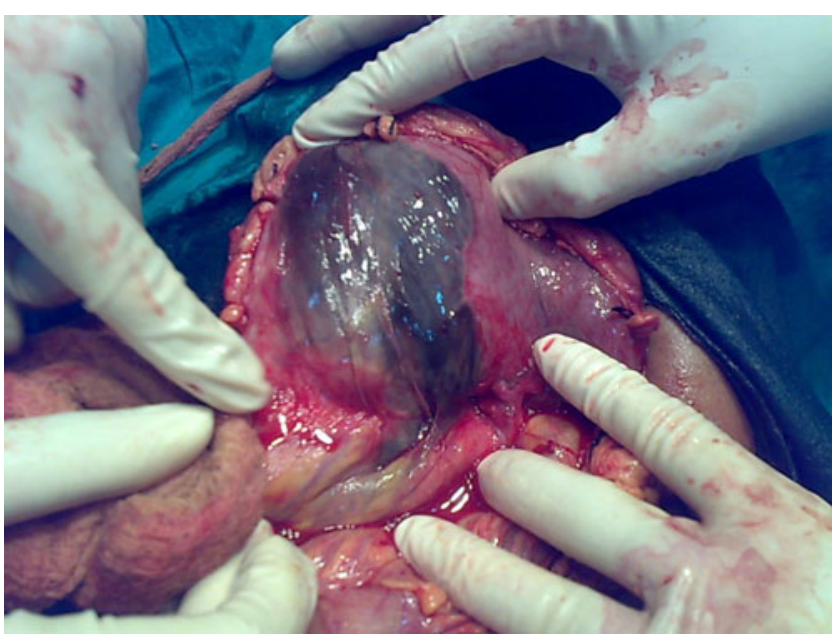

Fig. 1 Gross appearance of the stomach

\section{Discussion}

Gangrene of the stomach is a rare and fatal condition. Etiology includes thromboembolism and occlusion of major arterial supply, ingestion of corrosive agents, volvulus of the stomach, herniation of the stomach through the diaphragm, bulimia nervosa, iatrogenic gelfoam embolism, endoscopic haemostatic injections and infectious gastritis $[4,5]$. Other causes which have been associated with acute necrotizing gastritis and gangrene are recent large intake of alcohol, 'gastritis' and upper respiratory tract infections [2].

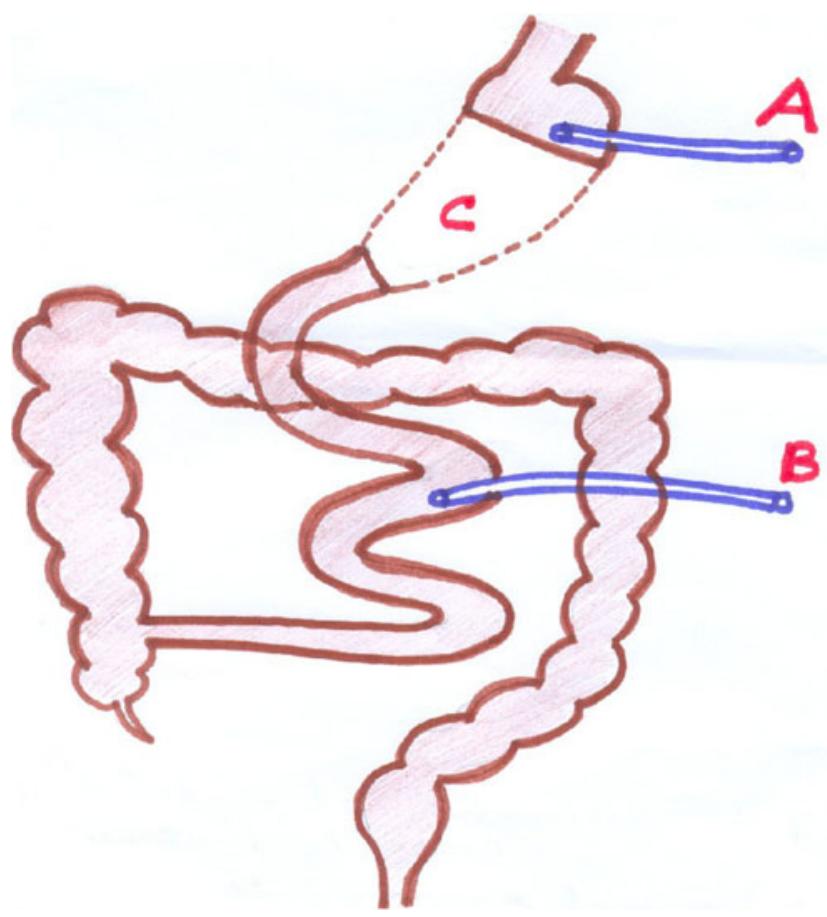

Fig. 2 schematic representation of emergency treatment a Proximal diversion tube $\mathbf{b}$ Feeding jejunostomy tube $\mathbf{c}$ Resected portion of the stomach

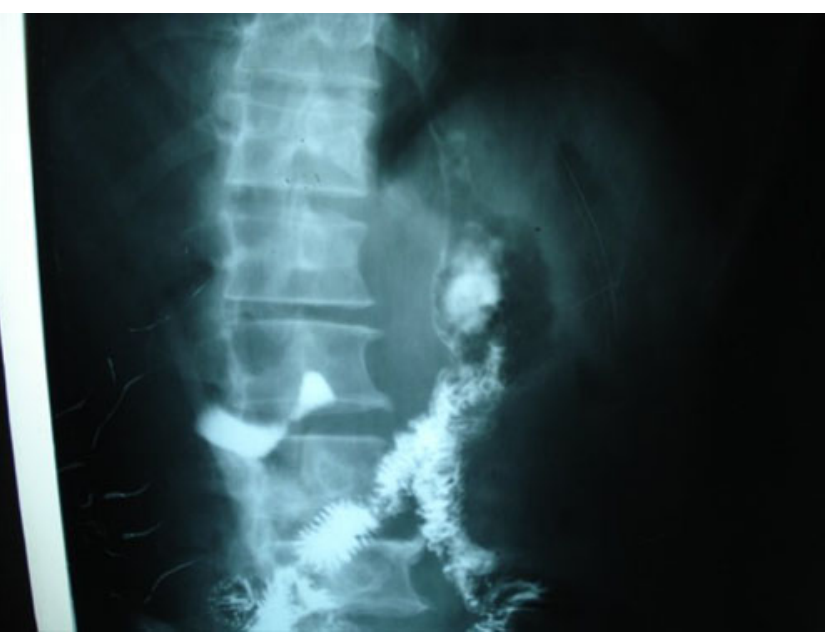

Fig. 3 Barium swallow showing the gastro-jejunal anastomosis

The infectious gastritis is subclassified into three forms including the suppurative, necrotizing and the emphysematous subtypes [3]. The acute necrotizing gastritis is the most rare cause of gastric gangrene. It begins as phlegmonous (suppurative) gastritis, then it progresses to the lethal severe form: acute necrotizing gastritis. Organisms isolated from the gastric wall include hemolytic streptococci, proteus, E. coli and clostridium welchii $[2,3]$.

In the present case there was no history suggestive of atherosclerosis. Neither volvulus nor herniation of the stomach was observed intraoperatively. The main vessels were intact, ruling out a thromboembolic event. There was no history of ingestion of caustic substances. There was generalized peritonitis that might have been caused by trans migration of organisms from the stomach to the peritoneal cavity [6]. Thus the possible cause of gangrene could be due to some necrotizing infection.

Diagnosis of gastric ischaemia is often delayed because of its rarity [7]. Initially, patients may have symptoms of mild epigastric tenderness, vomiting or diarrhoea that rapidly progresses to acute peritonitis, septic shock and death. All radiological tests are non specific. Gastroscopy may show purplish or blackish mucosa covered by exudates [3]. Absolute diagnosis is made, most frequently, at laparotomy $[8,9]$.

If a diagnosis of ischaemia is being considered, resuscitation and intravenous antibiotics should be initiated immediately, followed by an emergency exploratory laparotomy. Resection of a necrotic stomach is required, with total gastrectomy if necessary. One stage resection with esophago-jejunostomy has been reported [7]. Alternative includes resection with cervical esophagostomy for proximal diversion or resection and placement of an esophageal drain. A jejunal feeding tube should always be placed [7]. Diagnosis and treatment must be expeditious, because mortality rates for gastrectomy due to acute ischaemia are high. 


\section{Conclusion}

Gastric gangrene due to necrotizing gastritis is a rare and fatal disease. The diagnosis is usually made at laparotomy. Treatment consists of resection and feeding tube placement followed by intravenous antibiotics. Increased awareness of this rare entity may lead to more prompt diagnosis and an increased chance for patient survival [7].

\section{References}

1. Strauss RJ, Friedman M, Platt N, Gassner W, Wise L (1978) Gangarene of the stomach: A case of acute necrotizing gastritis. Am J Surg 135(2):253-7

2. Stein LB, Greenberg RE, Ilardi CF, Kurtz L (1989) Bank S. Am J Gastroenterol 84(12):1552-4
3. Hisham A R Hamad (2003) Post vagotomy gastric gangrene due to necrotizing gastritis. The Middle East Journal of Emergency Medicine 3(2), Sept 2003

4. Richieri JP, Pol B, Payan MJ (1998) Acute necrotizing ischemic gastritis: clinical, endoscopic and histopathologic aspects. Gastrointest Endosc 48:210-12

5. Dharap SB, Ghag G, Biswas A (2003) Acute necrotizing gastritis. Ind J Gastroenterol 22:150-51

6. Malhotra N, Singla SL, Marwah S, Goel R, Marwah N, Taneja R (2008) Gangrenous Perforation Of Stomach. The Internet Journal of Anaesthesiology 16(2)

7. Ammori GB, McHugh J, Cimmino VM (2007) Acute gastric necrosis: assessing the risk factors. Surgical Rounds. March 2007

8. Harvey RL, Doberneck RC (1972) Black WC:Infarction of the stomach following atheromatous embolization. Gastroenterology 62:469

9. Vilardel F (1985) Gastritis. Inc: Berk JE, Haubrich WS, Kalser MH, (eds.). Bockus gastroenterology. Philadelphia: WB Saunders, 943-4 